\title{
Review of: "Right fronto-parietal networks mediate the neurocognitive benefits of enriched environments."
}

\author{
Jiahui Deng ${ }^{1}$, Xiao Lin $^{2}$ \\ 1 Beijing Medical University \\ 2 Peking University
}

Potential competing interests: The author(s) declared that no potential competing interests exist.

Jiahui Deng ${ }^{1}$, Xiao Lin $^{1}$

1 Peking University Sixth Hospital, Peking University Institute of Mental Health, NHC Key Laboratory of Mental Health (Peking University), National Clinical Research Center for Mental Disorders (Peking University Sixth Hospital), Beijing 100191, China

Considering the robust impact of enriched environments on brain structure, and the elusive neurobiological basis underlying this relationship, this study tried to investigate the relationship between enriched environments, attentional capacity, and brain structural changes in older adults. The authors found that the fiber coherence of right superior longitudinal fasciculus mediated the relationship between enriched environment and brain atrophy, as well as the relationship between enriched environment and attention capacity. Although the study is generally well conducted, the manuscript also produces several major concerns which limit its accessibility or merit:

1. The operational definition of enriched environment is confusing. In the seminal study, Nucci proposed that the Cognitive Reserve Index questionnaire measures the cognitive reserve in aging clinical research. It is not plausible to use it as an index of enriched environment. It measures cognitive function in aging people, and attention belongs to a similar cognitive domain, which raise the question of circular argument.

2. In the Statistical Analyses section, the author conducted several independent models using several related factors, such as the scores of subscales, it is crucial to conduct multiple comparison correction;

3. When analyzing the relationship between ODI ( the fiber coherence) and gray matter volume, dose the authors have normalized the ODI to brain size. And dose the authors have a more precise hypothesis. For example, the volume of hippocampus and prefrontal cortex, which are related to cognitive function, could be a potential region of interested.

4. According to the data analysis method, the authors have a strong hypothesis on the direction of the 
relationships between four factors (EE, attention, Fiber coherence, and brain volume). It is important to re-analysis these data to exclude the opposed direction on the mediated effect.

5. The authors reported greater EE was associated with lower ODI, this results should be corrected for multiple comparison correction, since they also analyzed the relationship between EE and density of this particular fiber.

6. There are too many involved factory in this study, the authors could only focus on the relationship between white mater fiber coherence, brain structure and cognitive function, since the effect of EE on human is with a wide range. In the causal mediation models, is there direct relationship between EE with brain atrophy, and between EE with attention, if so, please re-draw figure 5.

Several minor issues to consider

1. A definition of the "enriched environment" in the introduction would be helpful for a better understanding. The EE seems to be the most predominant factor, and a precise definition is needed to clarify the confusion.

2. Please provide some references using the Cognitive Reserve Index as a definition of enriched environment.

3. In Figure 2, please provide a detailed description on the data analysis pipeline.

4. In Figure 4, please provide the $r$ and corrected $p$ values. 